\title{
Intérêt thérapeutique des facteurs de croissance granulocyto-monocytaires
}

Les CSF (colony-stimulating-factors) sont des protéines, facteurs de croissance des cellules hématopoïétiques. Les facteurs humains sont ou seront tous disponibles grâce aux méthodes des recombinants d'ADN. D'une remarquable efficacité biologique, ces substances pourraient bien modifier totalement l'approche thérapeutique des hypoplasies et des aplasies médullaires, primitives, toxiques, thérapeutiques ou infectieuses.

\section{Philippe Solal-Céligny Praticien hospitalo-universi- taire}

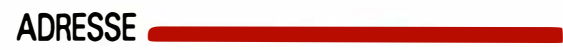

Ph. Solal-Céligny : service d'hématologie biologique, hôpital Antoine-Béclère, 157, rue de la Porte-de-Trivaux, 92140 Clamart, France.

$\mathrm{m} / \mathrm{s} n^{\circ} 4$ vol. 4, auril 88

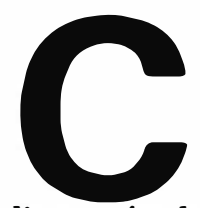

omme nombre d'activités biologiques de l'organisme, l'hématopoï̀se est sous la dépendance d'une régulation hormonale, permettant le maintien d'une production cellulaire stable à l'état basal, et son adaptation aux besoins éventuels. Cette régulation hormonale est complexe, associant des facteurs inducteurs ou facteurs de croissance et des facteurs inhibiteurs. Développées il y a près d'une vingtaine d'années, les techniques de culture in vitro des précurseurs médullaires de l'hématopoïèse ont permis d'identifier certains des facteurs inducteurs et de les purifier en quantités infinitésimales. A présent, grâce aux techniques de génie génétique, ces facteurs peuvent être produits en quantités suffisantes pour autoriser leur utilisation in vivo au cours de certaines anomalies primitives ou secondaires de l'hématopoïèse. Certaines indications cliniques de ces substances sont maintenant clairement établies, d'autres sont plus hypothétiques mais font de ces facteurs de croissance une arme thérapeutique en hématocancérologie, arme peut-être déterminante dans les années à venir. Nous n'envisagerons ici que les indications thérapeuti- ques des facteurs de croissance granulocyto-monocytaires.

\section{Schéma \\ de l'hématopoièse}

Toutes les cellules du sang ont pour origine une cellule souche dite totipotente de la moelle. Cette cellule souche a une capacité double, d'une part, d'autorenouvellement pour maintenir un compartiment stable de cellules souches, d'autre part, de différenciation vers une cellule souche déterminée pour un nombre plus restreint de lignées sanguines. Bien qu'il existe chez l'homme de multiples arguments en faveur de l'existence d'une cellule souche totipotente, elle n'a. pu être isolée et caractérisée. Les cellules non lymphoïdes du sang proviennent d'une cellule souche multipotente CFU-GEMM (colony-forming unit-granuloc te, erythrocyte, monoc te, megakar oc te). La CFU-GEMM se différencie elle-même en cellulessouches d'une seule lignée sanguine: CFU-MK pour la lignée mégacaryocyto-plaquettaire, CFU-GM pour la lignee granulocyto-monocytaire, CFU-Eo pour la lignée éosinophile, BFU-E et CFU-E pour la lignée érythroïde. Il est à noter que, si l'on garde.le terme de cellules souches pour 
désigner ces précurseurs d'une lignée, la capacité d'autorenouvellement diminue rapidement au fur et à mesure de la différenciation depuis la cellule souche totipotente et devient virtuellement nulle au stade de CFU propre à une seule lignée. Les cellules souches CFU de chaque lignée se différencient ensuite vers les précurseurs identifiables sur un frottis de moelle par la cytologie classique. Différents facteurs inducteurs et inhibiteurs régulent la production de cellules matures à partir de la cellule souche unipotente. Les techniques de culture in vitro de ces précurseurs ont permis d'identifier certains des facteurs inducteurs, alors que la nature, l'origine, le rôle des facteurs inhibiteurs demeurent en grande partie inconnus. Les facteurs inducteurs agissent par deux mécanismes: ils augmentent le renouvellement des cellules souches et favorisent leur différenciation vers une ou plusieurs lignées (figure 1).

\section{Facteurs de croissance granulocyto-monocytaires}

Les cultures de moelle chez la souris puis chez l'homme ont permis d'identifier quatre facteurs de croissance (Tableau I) agissant sur la lignée granulocyto-monocytaire [1, 2]: le GM-CSF (granulocyte, monocyte-colony-stimulating-factor, le G-CSF (granulocyte-colony-stimulating-factor), le M-CSF (monocyte-colony-stimulating factor) et l'interleukine 3 (IL-3). Tous quatre ont pu être clonés et pourront éventuellement être utilisés en thérapeutique dans un avenir plus ou moins proche. Deux sont spécifiques d'une lignée, le G-CSF pour la lignée granuleuse, le M-CSF pour la lignée monocytaire. Le GMCSF et l'IL-3 favorisent la croissance de colonies de plusieurs types. On a donc proposé un schéma selon lequel GM-CSF et IL-3 agiraient sur une cellule souche multipotente, tandis que G-CSF et M-CSF agiraient plus en aval, sur une cellule souche déterminée pour une seule lignée (figure 1).

GM-CSF humain. Le GM-CSF humain est une glycoprotéine monomérique comportant 127 acides aminés. Le poids moléculaire de la protéine est de $15 \mathrm{kDa}^{*}$, mais, dans les cellules mammifères, il varie entre 15 et $32 \mathrm{kDa}$ selon le degré de glycosylation. Le GM-CSF humain ne diffère de celui du gibbon que par sept acides aminés tandis que le degré

* $k D a=$ kilodalton

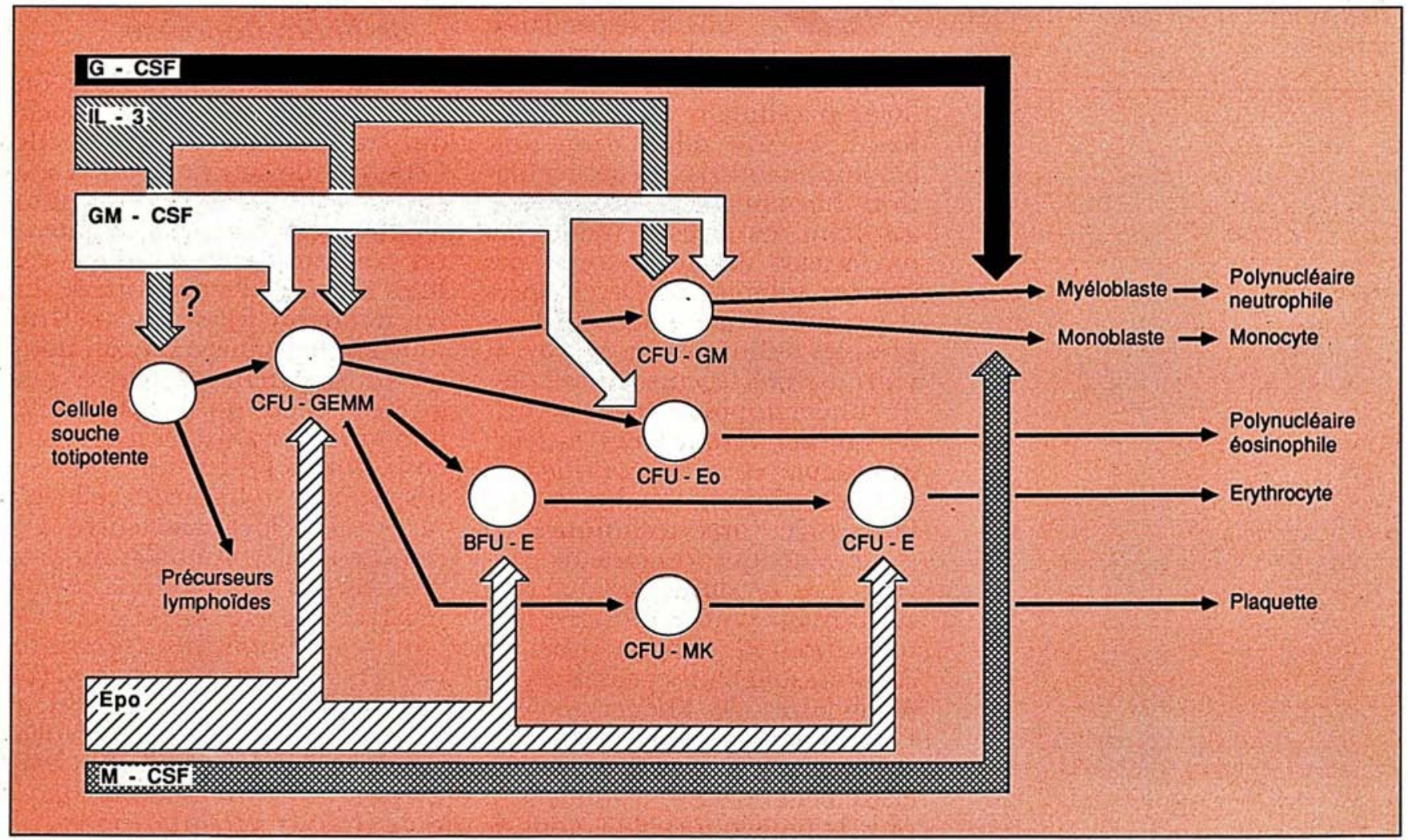

Figure 1. Schéma d'action de l'érythropoïétine et des facteurs de croissance granulocyto-monocytaires sur I'hématopö̈̈̀se. G-CSF = granulocyte-colony-stimulating factor ; IL-3 = interleukine 3; GM-CSF = granulocyte, monocytecolony-stimulating factor; CFU-GEMM = colony-forming unit-granulocyte, erythrocyte, monocyte, megakaryocyte; CFU-GM $=$ colony-forming unit-granulocyte-monocyte; CFU-Eo = colony-forming unit-eosinophils; BFU-E = burst-forming uniterythrocyte; CFU-E = colony-forming unit-erythrocyte ; CFU-MK = colony-forming unit-megakaryocyte ; Epo = érythropoïetine; $M-C S F=$ monocyte-colony-stimulating factor. 


\begin{tabular}{|c|c|c|c|c|c|}
\hline \multicolumn{6}{|c|}{$\begin{array}{c}\text { Tableau I } \\
\text { CARACTÉRISTIQUES DES FACTEURS DE CROISSANCE GRANULOCYTO-MONOCYTAIRES DE L'HOMME }\end{array}$} \\
\hline Nom . & Synonyme & $\begin{array}{l}\text { Poids moléculaire } \\
\text { (kDa) }{ }^{*}\end{array}$ & $\begin{array}{l}\text { Localisation } \\
\text { génomique }\end{array}$ & $\begin{array}{l}\text { Origines } \\
\text { cellulaires }\end{array}$ & $\begin{array}{l}\text { Lignées } \\
\text { cibles }\end{array}$ \\
\hline IL-3 & multi CSF & $20-26$ & $5 q$ & Lymphocytes $\mathrm{T}$ & $\begin{array}{l}\text { Neutrophiles } \\
\text { Monocytes } \\
\text { Eosinophiles } \\
\text { Basophiles } \\
\text { Erythrocytes } \\
\text { Mégacaryocytes }\end{array}$ \\
\hline GM-CSF & CSF- $\alpha$ & $14-35^{* *}$ & $5 q 21-q 32$ & $\begin{array}{l}\text { Lymphocytes T } \\
\text { Cellules } \\
\text { endothéliales } \\
\text { Fibroblastes }\end{array}$ & $\begin{array}{l}\text { Neutrophiles } \\
\text { Monocytes } \\
\text { Eosinophiles } \\
\text { Basophiles } \\
\text { Erythrocytes } \\
\text { Mégacaryocytes }\end{array}$ \\
\hline G-CSF & $C S F-\beta$ & $18-22$ & $17 q 11-q 21$ & $\begin{array}{l}\text { Monocytes } \\
\text { Fibroblastes }\end{array}$ & Neutrophiles \\
\hline M-CSF & CSF-1 & $35-45 \times 2$ & $5 q$ & $\begin{array}{l}\text { Monocytes } \\
\text { Fibroblastes } \\
\text { Cellules } \\
\text { endothéliales }\end{array}$ & Monocytes \\
\hline
\end{tabular}

* kilodalton; ** en fonction du degré de glycosylation. La nomenclature $q$ et $p$, utilisée pour les localisations chromosomiques, signifie : sur le grand bras (q), ou sur le petit bras (p).

d'homologie entre GM-CSF humain et murin n'est que de $54 \%$ [3]. L'origine cellulaire du GM-CSF est diverse: lymphocytes $\mathrm{T}$, fibroblastes et cellules endothéliales. La production et la sécrétion de GM-CSF sont ellesmême sous la dépendance de divers facteurs, notamment 2 cytokines d'origine monocytaire, l'interleukine l et le TNF $\alpha$ (tumor necrosis factor) [4] (figure 2, p. 132). Le GM-CSF est une multipoiétine, c'est-à-dire un facteur de croissance agissant sur plusieurs lignées, dans la mesure où il ne favorise pas seulement la croissance in vitro de colonies granulocyto-monocytaires mais également, en association avec l'érythropoïétine, de colonies érythroïdes et mixtes [5]. Il agit sur les lignées cibles par un récepteur spécifique, retrouvé sur les polynucléaires neutrophiles, éosinophiles et sur les monocytes. Ces récepteurs, qui ont pu être isolés, sont peu nombreux (50 à 250 par cellule) mais ont une affinité élevée $\left(\mathrm{Ka}=10^{8}\right.$ à $\left.10^{9} \mathrm{M}^{-1}\right)$ [6].
Interleukine 3. L'interleukine 3 a d'abord été isolée et purifiée chez la souris puis chez l'homme [7]. L'IL-3 humaine est une glycoprotéine dont le poids moléculaire de la forme recombinante varie entre 20 et $26 \mathrm{kDa}$ selon le degré de glycosylation. Les structures des IL-3 murine et humaine sont différentes; en revanche, il existe une grande homologie $(>99,5 \%)$ entre les formes de l'homme et du gibbon [7]. Toutes les origines cellulaires de l'IL-3 ne sont pas connues et seule la production par des lignées lymphoïdes $\mathrm{T}$ a été démontrée. Chez la souris comme chez l'homme, le gène de l'IL-3 est très proche de celui du GM-CSF, sur le chromosome 5 chez l'homme. L'interleukine 3 est une multipoïétine aux effets proches de ceux du GM-CSF. Chez la souris, l'interleukine 3 stimule la croissance des cellulessouches totipotentes mais cet effet n'a pas été démontré chez l'homme.

G-CSF. Le G-CSF a également été purifié chez la souris puis isolé, purifié et son gène a été cloné chez l'homme [8]. Son poids moléculaire est de $19 \mathrm{kDa}$ environ. Les cellules d'origine et les facteurs inducteurs, en particulier l'interleukine 1 et le TNF $\alpha$, sont identiques à ceux du GM-CSF. Son gène est situé sur le chromosome 17, au niveau du point de rupture intéressé dans les translocations $\mathrm{t}(15 ; 17)$ des leucémies aiguës promyélocytaires [9].

M-CSF (ou CSF-1). Chez la souris, le M-CSF isolé et purifié est un dimère fortement glycosylé, de poids moléculaire $70 \mathrm{kDa}$, chacune des 2 unités protéiques ayant un poids moléculaire de $14,5 \mathrm{kDa}$ [10]. Chez l'homme, le M-CSF, glycoprotéine également dimérique, est le produit d'un seul gène qui a pu être cloné et exprimé dans des cellules de mammifères [10]. Il existe peutêtre plusieurs protéines distinctes, bien qu'apparentées, de MCSF [10]. L'action du M-CSF semble limitée à la lignée monocytaire-macrophagique. Bien que cela reste à confirmer, il pourrait 


\section{RÉFÉRENCES}

1. Sieff CA. Hematopoietic growth factors. J Clin Invest $1987 ; 79$ : 1549-57.

2. Clarck SC, Kamen R. The human hematopoietic colony-stimulating factors. Science 1987 ; 236 : 1229-37.

3. Wong GG, Witek JS, Temple PA, et al. Human GM-CSF : molecular cloning of the complementary DNA and purification of the natural and recombinant proteins. Science $1985 ; 228: 810-5$

4. Vogel SN, Douches SD, Kaufman EN, Neta R. Induction of colony-stimulating factor in vivo by recombinant interleukin 1 and recombinant tumour necrosis factor $\alpha$. Immunol 1987 ; $138: 2143-8$.

5. Sieff CA, Emerson SG, Donahue RE, et al. Human recombinant granulocyte-macrophage colony-stimulating factor: a multilineage hematopoietin. Science 1985; 230 . $1171-3$

6. Gasson JC, Kaufman SE, Weisbart RH, Tomonaga M, Golde DW. High-affinity binding of granulocyte-macrophage colony-stimulating factor to normal and leukemic human myeloid cells. Proc Natl Acad Sci USA $1986 ; 83$ : 669-73.

7. Yang YC, Ciarletta AB, Temple PA, et al. Human Il-3 (multi-CSF): identification by expression cloning of a novel hemopoietic growth factor related to murine IL-3. Cell $1986 ; 47: 3-10$.

8. Souza L, Boone T, Gabrilove JL, et al. Recombinant human granulocyte colony-stimulating factor: effects on normal and leukemic myeloid cells. Science 1986 ; 232 : 61 -5.

9. Simmers RN, Webber LN, Shannon MF, et al. Localization of the G-CSF gene on chromosome 17 proximal to the breakpoint in the $t(15 ; 17)$ in acute promyelocytic leukemia. Blood $1987 ; 70$ : 330-2.

10. Wong GG, Temple PA, Leary AC, et al. Human CSF-1: molecular cloning and expression of 4-kb cDNA encoding the human urinary protein. Science $1987 ; 235$ : 1504-8.

11. Sherr CJ, Rettenmier CW,Sacca R, Roussel MF, Look AT, Stanley ER. The c-fms proto-oncogene product is related to the receptor for the mononuclear phagocyte avoir des actions indirectes sur d'autres lignées en provoquant la libération par les monocytes de cytokines, notamment l'IL-l et le TNF $\alpha$, qui à leur tour stimuleraient la production d'autres CSF (figure 2) [1]. Le nombre de récepteurs du M-CSF sur les cellulescibles est plus élevé que celui des autres facteurs de croissance. Ce récepteur est vraisemblablement identique ou très proche du produit, à activité tyrosine-kinase, de l'oncogène $c$-fms [11].

\section{Effets des traitements in vivo et in vitro}

Parmi les facteurs de croissance granulocyto-monocytaires, seuls deux sont parvenus au terme du stade pré-clinique de développement, et aux premiers essais cli- niques chez l'homme, le GM-CSF et le G-CSF. L'interleukine 3 et le M-CSF en sont au stade de l'étude in vitro et au début des expérimentations animales.

En culture in vitro de précurseurs médullaires, le GM-CSF et le GCSF humains obtenus par recombinaison génétique (abrégés respectivement en rhGM-CSF et rhG-CSF) permettent une stimulation, fonction de la dose de la croissance des précurseurs CFUGM $[8,12]$; en outre, le rhGMCSF est apparu, comme son équivalent naturel, favoriser la croissance des cellules souches érythroïdes et mégacaryocytoplaquettaires, et de ce fait, est considéré comme une multipoïétine [5].

Chez le primate, le rhGM-CSF injecté par voie intraveineuse

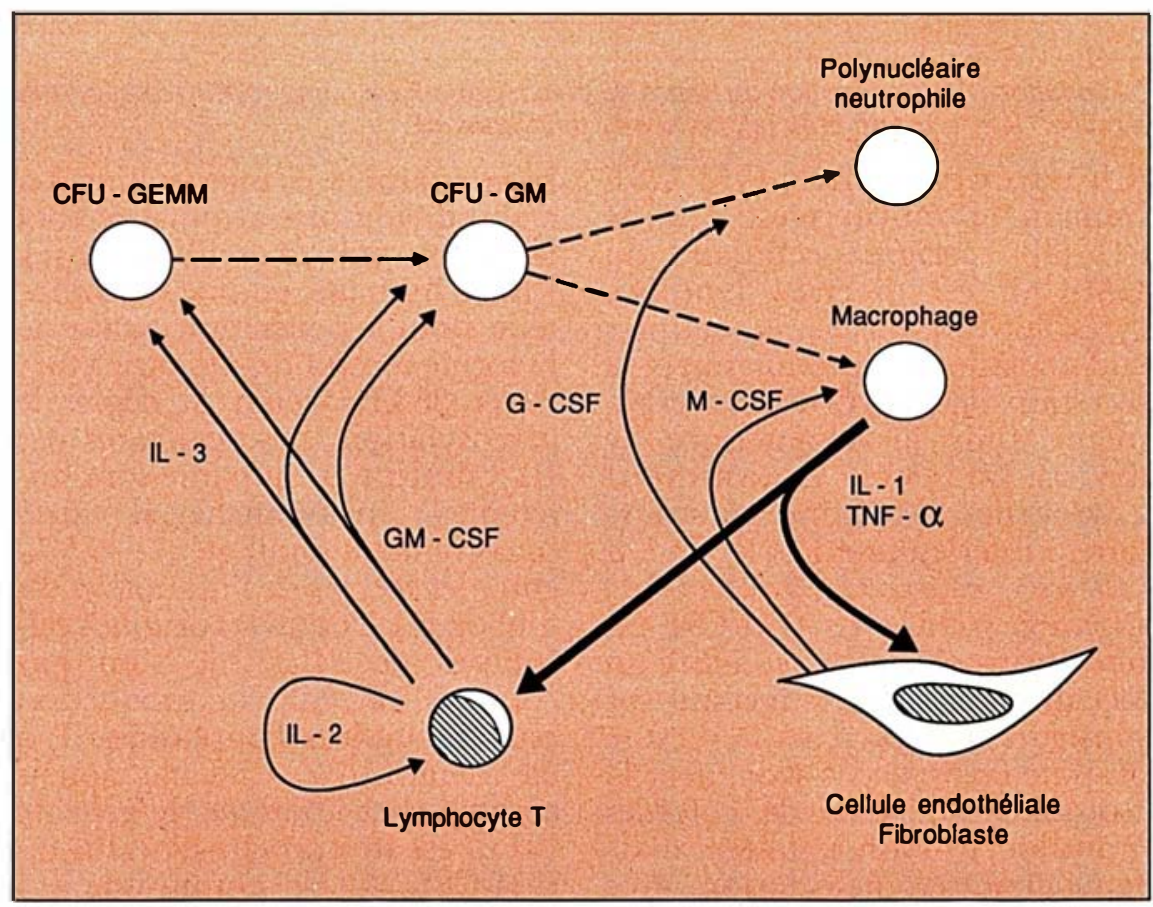

Figure 2. Schéma des interactions entre progéniteurs granulocyto-monocytaires, Iymphocytes et cellules endothéliales ou fibroblastes. Les macrophages activés libèrent de l'interleukine 1 (IL-1) et du TNF $\alpha$ (tumor necrosis factor) qui induisent une synthèse et une libération de facteurs de croissance granulocyto-monocytaires : interleukine 3 et GM-CSF par les lymphocytes Tactivés, G-CSF et M-CSF par les cellules endothéliales et les fibroblastes (et probablement aussi par les lymphocytes T). Interleukine 3, GM-CSF et M-CSF augmentent la production médullaire de monocytes; en outre, le M-CSF active les macrophages et induit la synthèse d'interleukine 1 et de TNF $\alpha$. II se crée ainsi une boucle autoentretenue dont les modalités de « freinage » ne sont pas connues. 
1. Aplasies médullaires globales ou électives

- Aplasie médullaire idiopathique

- Irradiations corporelles accidentelles

- Neutropénies chroniques constitutionnelles, cycliques ou non

- Neutropénies immunologiques : syndrome de Felty, leucémie lymphoïde chronique à cellules T suppressives

- Agranulocytoses aiguës immuno-allergiques d'origine médicamenteuse

2. Leuconeutropénies d'origine virale

- Infections à virus HIV

- Infections liées au virus d'Epstein-Barr

- Autres infections virales (groupe herpès)

3. Traitement de la toxicité médullaire de chimiothérapies antinéoplasiques

- Tumeurs solides : carcinomes ovariens, mammaires, testiculaires, sarcomes, etc.

- Hémopathies lymphoïdes : leucémies aiguës lymphoïdes, lymphomes non hodgkiniens

- Hémopathies myéloïdes : leucémies aiguës (?), syndromes myélodysplasiques

- Maladie de Hodgkin

- Allogreffes ou autogreffes de moelle

- Traitements par modificateurs de la réponse biologique : interféron $\alpha$, interféron $\gamma$, interleukine 2 , tumor necrosis factor

4. Infections bactériennes avec déficit quantitatif et/ou qualitatif des cellules granuleuses

- Brûlures étendues

- Carences et dénutrition

- Septicémies graves avec neutropénie (pneumocoques, méningocoques, Salmonella, etc.).

continue à la dose $50 \mathrm{U} / \mathrm{kg} / \mathrm{min}^{*}$ induit avec un délai de 24 heures, une hyperleucocytose qui atteint son maximum après 4 à 8 jours et régresse en 24 heures à l'arrêt de la perfusion. La leucocytose est multipliée par sept par accroissement des nombres de polynucléaires neutrophiles mais aussi de monocytes et d'éosinophiles (le nombre de ces derniers étant multiplié par 30) [13]. Les réticulocytes et les plaquettes sont également discrètement augmentés [13]. D'autres études ont montré que le rhGM-CSF par voie sous-cutanée avait une efficacité au moins égale à celle de la voie intra-veineuse [14]. Les formes non glycosylées de rhGM-CSF obtenues par expression du gène dans des bactéries $E$. coli sont apparues avoir la même efficacité que les formes glycosylées obte-

* Une unité de rhGM-CSF correspond à la quantité produisant une incorporation de thymidine tritiée égale à la moitié de l'incorporation maximale en culture de cellules granuleuses sanguines d'un sujet atteint de leucémie myéloïde chronique (méthode de Griffin).

$\mathrm{m} / \mathrm{s} n^{\circ} 4$ vol. 4 , auril 88 nues par expression du gène dans des cellules de mammifères, ce qui pourrait ultérieurement simplifier les processus de fabrication [14].

Le G-CSF, à la dose de $10 \mu \mathrm{g}$ / $\mathrm{kg} / \mathrm{j}$ par voie sous-cutanée induit une hyperleucocytose fonction de la dose, avec une cinétique voisine de celle du GM-CSF [15]. L'augmentation porte sur les neutrophiles $(\times 10$ à 30$)$ et à un moindre degré sur les lymphocytes $(\times 1,5$ à 2,5$)$, tandis que les nombres absolus de monocytes et de polynucléaires éosinophiles ne varient pas significativement [15]. Sous traitement par rhGM-CSF ou rhG-CSF, une importante hyperplasie, prédominant sur la lignée granuleuse [13, 15], observée sur les frottis de moelle, confirme l'augmentation de la production cellulaire.

Diverses études in vitro ont montré, qu'outre leurs effets quantitatifs sur la production médullaire de cellules granuleuses, le rhGMCSF et le rhG-CSF avaient des effets qualitatifs sur les cellules produites. Ainsi, le rhGM-CSF : (a) inhibe la mobilité des neutrophiles et favorise leur maintien dans un site inflammatoire; (b) augmente la production d'anions superoxydes en réponse à des peptides activateurs ( $\mathrm{N}$-formyl-méthionyl-leucyl-phénylalanine) [16]; (c) accroît, en présence d'anticorps, la cytotoxicité des monocytes indépendamment de tout autre stimulus exogène [17]; (d) favorise les liaisons interleucocytaires en augmentant l'expression membranaire des molécules d'adhésion [18]; (e) accroît la phagocytose et la destruction de bactéries [19].

L'augmentation de la production d'anions superoxydes en réponse à un stimulus et de la bactéricidie s'observe in vivo chez le primate traîté par rhGM-CSF [14]. In vivo également, le rhG-CSF accroît la réduction du nitrobleu de tétrazolium [15].

\section{Indications}

Le développement clinique des formes recombinantes des facteurs de croissance granulocyto-mono- 


\section{RÉFÉRENCES}

12. Metcalf D, Begley CG, Johnson GR, et al. Biological properties in vitro of a recombinant human granulocyte-macrophage colony-stimulating factor. Blood 1986; 67 : 37-45.

13. Donahue RE, Wang EA, Stone DK, et al. Stimulation of hematopoiesis in primates by continuous infusion of recombinant human GM-CSF Nature $1986 ; 321: 872-5$.

14. Mayer P, Lam C, Obenaus H. Liehl E, Besemer J. Recombinant human GM-CSF induces leukocytosis and activates peripheral blood polymorphonuclear neutrophils in non-human primates. Blood 1987 ; 70 : 206-13.

15. Welte K, Bonilla MA, Gillio AP, et al. Recombinant human granulocyte colony-stimulating factor. Effect on hematopoiesis in normal and cyclophosphamide treated primates. J Exp Med 1987; 165 : 941-8.

16. Weisbart RH, Golde DW, Clark SC, Wong GG, Gasson JC. Human granulocyte-macrophage colony-stimulating factor is a neutrophil activator. Nature 1985 ; 314 : 361-3.

17. Grabstein KH, Ordal DL, Tushinski RJ, et al. Induction of macrophage tumoricidal activity by granulocyte-macrophage colonystimulating factor. Science 1986 ; 236 : 506-8.

18. Arnaout MA, Wang EA, Clark SC, Sieff CA. Human recombinant granulocytemacrophage colony-stimulating factor increases cell-to-cell adhesion and surface expression of adhesion-promoting surface glycoprotein on mature granulocytes. J Clin Invest $1986 ; 78: 597-601$.

19. Fleischmann J, Golde DW, Weisbart RH, Gasson JC. Granulocyte-macrophage colonystimulating factor enhances phagocytosis of bacteria by human neutrophils. Blood 1986 : 68 : 708-11.

20. Antin JH, Smith BR, Rosenthal DS, Holmes W, Bonn HF. Phase I/II study of recombinant human granulocyte-macrophage colony-stimulating factor (GM-CSF) in bone marrow failure. Blood 1987; 70 (suppl. 1) : 372A.

21. Donahue RE, Seehra J. Norton C, et al. Stimulation of hematopoiesis in primates with human interleukin 3 and granulocytemacrophage colony-stimulating factor. Blood 1987 : 70 (suppl. 1) : 388A.

22. Nienhuis AW, Donahue RE, Karlsson S, et al. Recombinant human granulocytemacrophage colony-stimulating factor (GMCSF) shortens the period of neutropenia after autologous bone marrow transplantation in a primate model. J Clin Invest 1987 ; 80 : 573-7.

23. Morstyn G, Duhrsen U, Campbell L, et al. Granulocyte colony-stimulating factor (GCSF) in patients with advanced cancer receiving melphalan. Blood 1987; 70 (suppl. 1) : cytaires (que nous regrouperons sous la forme abrégée de rh-CSF) n'en est qu'à son tout début. Les indications que nous allons détailler ne sont donc que des orientations proposées pour ce développement: certaines disparaîtront pour des raisons d'inefficacité, de toxicité ou de coût, d'autres pourront se révéler à l'usage.

En outre, les essais cliniques du rhG-CSF et du rhGM-CSF en sont à leur début, tandis que le rhIL-3 et le rhM-CSF ne seront disponibles qu'à moyen terme. Il est actuellement presque impossible de séparer les indications selon le facteur de croissance. Tout juste peut-on mentionner, pour certaines indications, qu'une multipoïétine (rhGMCSF ou rhIL-3) semble, a priori, devoir être préférée à un facteur de croissance n'agissant que sur une seule lignée. Les principales indications potentielles des rh-CSF (Tableau II, page 135) peuvent être séparées en: (1) hypoplasies primitives ou secondaires de la moelle osseuse; (2) prévention et traitement de la toxicité myéloïde des agents anti-cancéreux; (3) hémopathies malignes; (4) infections virales et bactériennes.

Les aplasies médullaires globales ou limitées à la lignée granuleuse (neutropénies chroniques, agranulocytoses immuno-allergiques, syndrome de Felty*, etc.) relèvent d'un mécanisme immunitaire ou de lésions toxiques directes des cellules souches. L'efficacité des rh-CSF dans les aplasies toxiques dépendra de l'intensité de la destruction du compartiment des cellules souches. Dans le cas des aplasies ou des hypoplasies médullaires à médiation immunitaire, le traitement par rh-CSF devra probablement être associé à un traitement immuno-suppresseur pour permettre aux cellules souches stimulées de produire des cellules matures.

\footnotetext{
* Syndrome de Felty: association d'une polyarthrite rhumatoïde, d'une splénomégalie et d'une neutropénie.
}

Les premiers essais cliniques confirment l'efficacité des rh-CSF dans ces affections. Ainsi, chez six patients atteints d'aplasie médullaire idiopathique, le rhGM-CSF administré pendant 21 jours a permis une augmentation du nombre de polynucléaires neutrophiles $(X 1,5$ à $\times 3,5)$ et de monocytes (X 14 à $X$ 28) [20]. Cette amélioration ne persiste cependant pas à l'arrêt du traitement. L'aplasie médullaire représente probablement un exemple d'affection justifiant des traitements associant plusieurs facteurs de croissance agissant sur des cellules souches de degrés de maturation différents. La meilleure efficacité chez le singe macaque rendu aplasique est obtenue par un traitement comportant successivement de la rh-IL3 puis du rhGM-CSF [21].

Les radiothérapies étendues et les polychimiothérapies intensives sont responsables d'une toxicité médullaire qui prédomine le plus souvent sur la lignée granuleuse. Cette toxicité, principale cause de morbidité de ces traitements, limite parfois les doses que l'on peut administrer et surtout explique les délais à respecter entre les cycles de traitement. Le traitement préventif et curatif de cette toxicité médullaire sera probablement une indication privilégiée des facteurs de croissance rhGMCSF et rhG-CSF comme en témoignent les premiers travaux publiés chez l'animal et chez l'homme. Ainsi, chez le primate traité par cyclophosphamide à fortes doses, la durée de l'agranulocytose induite est supérieure à quatre semaines chez l'animal ne recevant aucun facteur de croissance et raccourcie à quatre-cinq jours chez l'animal traité par rhG-CSF dès le début du traitement cytotoxique [15]. Un raccourcissement aussi spectaculaire de l'agranulocytose a été observé grâce à un traitement par rhGMCSF chez des primates rendus aplasiques par la cyclophosphamide associée à une irradiation corporelle totale et suivie d'une autogreffe de moelle [22].

Plusieurs essais cliniques phases 
I/II chez l'homme ont confirmé que le rhGM-CSF ou le rhG-CSF permettraient de raccourcir sensiblement la durée de la neutropénie après chimiothérapie antinéoplasique (melphalan à fortes doses [23], polychimiothérapie pour sarcome [24] ou pour carcinome vésical à cellules transitionnelles [25]).

Néanmoins, avant tout essai des facteurs de croissance granulocyto-monocytaires au cours des néoplasies, il faut s'assurer, par des tests in vitro sur des lignées néoplasiques, qu'ils ne constituent pas un facteur favorisant la croissance de ces néoplasies. Ainsi, le GM-CSF est un facteur de croissance de lignées de carcinomes microcellulaires d'origine broncho-pulmonaire [25]; en revanche, il ne favorise pas la croissance de lignées lymphomateuses [26]. Dans le premier cas, l'utilisation du GM-CSF expose au risque de favoriser la croissance tumorale.

Les traitements par rhG-CSF et/ou rhGM-CSF (et/ou peut-être rhIL-3) devraient entraîner de profondes modifications des concepts actuels de la chimiothérapie antinéoplasique. Les doses de certains antimitotiques pourront être augmentées pour surmonter certaines résistances ou, plus probablement, l'espace entre les cycles et la durée totale du traitement seront raccourcis. Les rh-CSF permettront de diminuer très sensiblement la morbidité des chimiothérapies antinéoplasiques les plus lourdes.

Les traitements à visée anti-cancéreuse par les substances rassemblées sous le nom de modificateurs de la réponse biologique (interférons $\alpha$ et $\gamma$, interleukine 2, TNF $\alpha$ ) sont également responsables d'une toxicité médullaire, qui constitue l'un des facteurs limitant leur utilisation. In vitro, l'inhibition par les interférons de la pousse des progéniteurs CFUGM est levée par le GM-CSF. Le rhGM-CSF et/ou le rhG-CSF permettront peut-être ainsi de prévenir la toxicité hématopoïétique des modificateurs de la réponse biologique.

$m / s n^{\circ} 4$ vol. 4, avril 88
L'utilisation du rhGM-CSF et/ou du rhG-CSF au cours des hémopathies malignes pose des problèmes différents selon le type cytologique. Au cours des hémopathies lymphoïdes, ils pourront permettre de raccourcir la durée de la cytopénie après polychimiothérapie, auto- ou allogreffe.

En revanche, leur prescription au cours des hémopathies myéloïdes, notamment des leucémies aiguës myéloïdes ou des syndromes myélodysplasiques, nécessitera au préalable de multiples études in vitro. En effet, les facteurs de croissance granulocyto-monocytaires ne sont pas dépourvus d'action sur les précurseurs leucémiques clonaux.

- D'une part, le rhGM-CSF et le rhG-CSF favorisent in vitro la croissance de clones de cellules leucémiques myéloïdes [8, 27, 29]. L'administration de rhGM-CSF ou de rhG-CSF au cours d'une hémopathie myéloïde pourrait donc aggraver l'évolution de la leucémie. Cependant, la stimulation in vitro de la croissance des progéniteurs blastiques par le rhGM-CSF et le rhG-CSF est très variable d'un cas à l'autre, nulle dans certains [27, 29].

- D'autre part, les études sur des lignées leucémiques [30] ou sur cellules leucémiques fraîches [27] ont montré que le rhGM-CSF ou le rhG-CSF favorisaient la différenciation des cellules leucémiques comme en témoignaient des modifications morphologiques ou l'acquisition de capacités fonctionnelles propres aux cellules granuleuses ou monocytaires matures [29].

En définitive, l'action thérapeutique (ou au contraire, délétère) des rhGM-CSF ou rhG-CSF au cours d'une leucémie aiguë myéloïde ou d'un syndrome myélodysplasique sera la résultante, variable selon les cas: (a) de l'éventuelle stimulation de la croissance du clone blastique; (b) de l'action différenciatrice sur les cellules blastiques; (c) des effets sur d'éventuels progéniteurs normaux résiduels.

Un essai clinique préliminaire a montré une action potentielle- ment intéressante des rhCSF au cours de syndromes myélodysplasiques. Un traitement par rhGMCSF a permis d'augmenter significativement les nombres de polynucléaires neutrophiles et de monocytes du sang chez huit malades atteints de syndrome myélodysplasique (anémie réfractaire avec excès de blastes, en transformation ou non). La diminution de la dysplasie hématopoïétique, du degré d'infiltration blastique et la présence d'anomalies cytogénétiques dans les cellules matures produites indiquent que le rhGM-CSF a probablement favorisé la différenciation des cellules clonales immatures [31].

Les cytopénies granuleuses sont fréquentes au cours des infections à virus HIV (virus du syndrome d'immuno-déficience acquise). Leurs mécanismes sont multiples: présence d'auto-anticorps et, surtout, infection des précurseurs médullaires par le virus HIV suivie d'un effet cytopathique. Ces cytopénies sont souvent aggravées par certains médicaments (cotrimazole, azidothymidine). Au cours d'un essai phase I/II chez des malades atteints de SIDA et ayant une leuconeutropénie, le rhGM-CSF a permis de rapidement corriger la neutropénie et l'amélioration persista tout au long des 14 jours de traitement [32]. Dans un essai in vitro, les effets métaboliques de l'azidothymidine (AZT) sur les cellules mononucléées du sang (déplétion en nucléotides triphosphates) étaient diminués par le rhGMCSF [33]. Le rhGM-CSF pourrait donc diminuer la toxicite médullaire de l'AZT.

En outre, une étude préliminaire [34] a montré qu'in vitro le rhGM-CSF diminuait l'activité «transcriptase reverse», témoin de la réplication virale, dans une lignée monocytaire continue (U937) infectée par le virus HIV. Une action directe du GM-CSF sur l'infection par le virus HIV n'est donc pas à exclure.

$\mathrm{Au}$ cours de certaines infections bactériennes, le rhGM-CSF ou le rhG-CSF pourront peut-être 


\section{RÉFÉRENCES}

24. Antman K, Griffin J, Elias A, et al. Use of rGM-CSF to ameliorate chemotherapy induced myelosuppression in sarcoma patients. Blood 1987; 70 (suppl. 1) : 394 A.

25. Baldwin GC, Di Pierso J, Kaufman SE, Quan SG, Golde DW, Gasson JC. Characterization of human GM-CSF receptors on nonhematopoietic cells. Blood 1987; 70 (suppl. 1): 519A

26. Wilson W, Robin M, Mechinaud F, et al. The effect of recombinant human GM-CSF on the proliferation of human B-cell lymphomas. Blood 1987 ; 70 (suppl. 1) : 434A.

27. Hoang $T$, Nara $N$, Wong $G$, Clark $S$ Minden MD, Mc Culloch EA. E.ffects of recombinant GM-CSF on the blast cells of acute myeloblastic leukemia. Blood 1986; 68 313-6.

28. Kelleher C, Miyauchi J, Wong G. Clark $S$, Minden MD, Mc Culloch EA. Synergism between recombinant growth factors, GMCSF and G-CSF acting on the blast cells of acute myeloblastic leukemia. Blood 1987 ; 69 . 1498-503.

29. Miyauchi J, Kelleher CA, Yang YC, et al. The effects of three recombinant growthfactors, IL-3, GM-CSF and G-CSF on the blast cells of acute myeloblastic leukemia maintained in short-term suspension culture. Blood 1987 ; 70 : 657-63.

30. Begley CG, Metcalf D, Nicola NA. Purified colony stimulating factors (G-CSF and GM-CSF) induce differentiation in human HL60 leukemic cells with suppression of clonogenicity. Int J Cancer 1987; 39 : 99-105.

31. Vadhan-Raj S, Keating M, Le Maistre A, et al. Effects of recombinant human granulocyte-macrophage colony-stimulating factor in patients with myelodysplastic syndromes. $N$ Engl J Med 1987 ; 317 : 545-52.

32. Groopman J, Mitsuyasu RT, Deleo MJ, Oette DH, Golde DW. Effect of recombinan human granulocyte-macrophage colony-stimulating factor on myelopoiesis in the acquired immunodeficiency syndrome. $N$ Engl J Med $1987 ; 137: 593-8$

33. Birkhofer M, Grants-Graham G, Bhalla K. Effect of recombinant human granulocyte/macrophage colony stimulating factor (GM-CSF) on '3'-azido-3' deoxythymidine (AZT) mediated depletion of deoxyribonucleotide pools in normal human bone marrow mononuclear cells. Blood 1987 ; 70 (suppl. 1) : 376A.

34. Hammer SM, Gillis JM, Groopman JE Rose RM. In vitro modification of human immunodeficiency virus infection by granulocyte-macrophage colony-stimulating factor and $\gamma$ interferon. Proc Natl Acad Sci USA $1986 ; 83 ; 8734-38$. accroître la réponse leucocytaire, améliorer les capacités bactéricides des cellules granuleuses et monocytaires et favoriser ainsi la guérison. Ainsi, un traitement par rhGM-CSF ou par rhG-CSF pourra s'envisager au cours: (a) des infections des grands brûlés, qui s'accompagnent souvent de leuconeutropénie; (b) de certaines formes graves de pneumococcémie avec agranulocytose; (c) des infections sévères de sujets débilités (dénutrition, carences, etc.) chez qui la réponse granuleuse est quantitativement et qualitativement insuffisante.

Certes le nombre des malades traités par rhGM-CSF ou rhG-CSF est encore très faible. Les effets indésirables de ces facteurs de croissance apparaissent néanmoins limités (douleurs osseuses, syndrome œedémateux aux posologies les plus élevées, quelques accès fébriles), en tout cas, beaucoup moins sévères que ceux observés au cours des essais des autres modificateurs de la réponse biologique (interférons, interleukine 2). Des effets secondaires plus préoccupants ne sont pas à exclure et pourront s'observer au cours de traitements plus prolongés ou dans d'autres indications. Ainsi, il est possible que l'hyperéosinophilie majeure induite par le rhGM-CSF ait une toxicité cardiaque ou pulmonaire si elle est prolongée. Au cours des infections, notamment avec localisations pulmonaires, la production brutale sous l'influence de rhGMCSF ou de rhG-CSF de phagocytes activés est potentiellement susceptible de provoquer ou d'aggraver un œdème pulmonaire lésionnel.

Les indications potentielles des facteurs de croissance granulocyto-monocytaires apparaissent multiples et le traitement de certaines affections aussi fréquentes que le cancer sera probablement modifié en profondeur par leur mise à la disposition du thérapeute. Néanmoins, la rigueur et la prudence dans le développement s'imposent pour éviter de trop brutales désillusions après de trop aveugles espoirs

\section{Summary}

Several growth factors stimulate the granulopoiesis: interleukin 3 (IL-3) and granulocyte, monocyte-colonystimulating factor (GM-CSF) act on pluripotent cells and immature progenitor cells; granulocyte-colony-stimulating factor (G-CSF) and macrophage-colony-stimulating factor (M-CSF) act on more mature progenitor cells. The genes for these hormones have been cloned and expressed and large amounts have become available for therapeutic use. After reviewing the basics of granulocytic growth factor physiology, the author details the potential indications of these factors : primary deficiencies of bone marrow production; prevention and treatment of the myeloid toxicity of anticancer treatments; cure of myeloid disorders induced by some viruses especially Human Immunodeficiency Virus and Epstein-Barr virus : severe infections in debilitated patients.

\section{TIRÉS A PART}

Ph. Solal-Céligny : service d'hématologie biologique, hôpital Antoine-Béclère, 157, rue de la Porte-de-Trivaux, 92140 Clamart, France. 